Discussion Paper No. 832

\title{
OPTIMAL OBSCURITY IN THE ACQUISITION AND DISCLOSURE OF INFORMATION ABOUT A SHOCK
}

\author{
Masaki Aoyagi
}

February 2012

The Institute of Social and Economic Research

Osaka University

6-1 Mihogaoka, Ibaraki, Osaka 567-0047, Japan 


\title{
Optimal Obscurity in the Acquisition and Disclosure of Information about a Shock
}

\author{
Masaki Aoyagi* \\ Osaka University
}

February 15, 2012

\begin{abstract}
A principal acquires information about a shock and then discloses it to an agent. After the disclosure, the principal and agent each decide whether to take costly preparatory actions that yield benefits only when the shock strikes. The principal maximizes his expected payoff by controlling the quality of his information, and the disclosure rule. We show that even when the acquisition of perfect information is costless, the principal may optimally acquire imperfect information when his own action eliminates the agent's incentive to take action against the risk.

Key words: endogenous information, disclosure, signal quality, transparency, specific investment, strategic ignorance.

Journal of Economic Literature Classification Numbers: C72, D82.
\end{abstract}

*ISER, Osaka University, 6-1 Mihogaoka, Ibaraki, Osaka 567-0047, Japan. 


\section{Introduction}

Preparing for a variety of natural, social, and economic shocks is an important task of every government. Many governments appropriate a large amount of money on research into the forecasting of such natural shocks as hurricanes, snow storms and other extreme weather conditions, earthquakes, epidemic outbreaks, and so on. ${ }^{1}$

Along with forecasting, a government's strategies to prepare for those shocks typically involve two forms of interventions. The first is a direct intervention that is implemented at the government's own cost. The second is an indirect intervention that consists of raising public awareness of the risk of the shocks and advising the public to take preparatory actions themselves. In the case of an epidemic outbreak, for example, the direct interventions include stricter quarantine control, building depressurized rooms at hospitals, increasing the stock of anti-virus medicines, and so on. On the other hand, an indirect intervention consists of advice to the public to receive vaccinations, avoid traveling and exercise hygiene practices. Likewise, against earthquakes, direct interventions include enforcing stricter building codes and reinforcing public buildings such as schools and highways, while indirect interventions include advice to the public to reinforce their own houses, prepare food stocks, and purchase earthquake insurance. Unlike direct interventions, it is the public themselves who bear the cost of the advised action. ${ }^{2}$ The same story also applies to economic shocks such as currency crises, food and energy price hikes, and market crashes. Governments engage resources in the forecasting of such shocks, and prepare for them by intervening directly by adjusting portfolios, building food and oil stocks, and subsidizing the development of alternative technologies. The essential feature of many of these preparatory actions is that they are specific investment in the sense that they have value only when the shock strikes.

It is argued by some that the policy of spending much money on forecasting

\footnotetext{
${ }^{1}$ For example, National Oceanic and Atmospheric Agency (NOAA) of the United States budgeted more than $\$ 2,000$ million on weather services and satellites. Its joint polar satellite system (JPSS), which is used for mid-range weather forecasts, alone cost US\$382 million in FY2010 ("NOAA warns weather forecasts will suffer from budget cuts," Washington Post 03/31/2011). As another example, the US Geological Survey budgeted more than US $\$ 90$ million for research into geologic hazard assessments in FY2010.

${ }^{2}$ Skoufias (2003) discusses the strategies employed by households and public agencies to mitigate the damages of economic crises and natural disasters. Some indirect interventions involve public expenditure as in the case of subsidies for vaccination programs, or those for the installation of solar panels.
} 
shocks and at the same time advising the public to take preparatory measures is inconsistent. ${ }^{3}$ One interpretation of this claim is as follows: If the accurate forecasting of a shock is possible, then the public is led to think that timely direct interventions will save them costly efforts. On the other hand, from the point of view of the government, indirect interventions are much less costly and the public's own action is often more effective in mitigating the damage.

The purpose of this paper is to provide a formal justification of the above logic in the framework of information acquisition by a principal (government) and its disclosure to an agent (the public). We show that acquiring perfect information may indeed be suboptimal when the agent may free-ride on the principal's effort. A more detailed description of our model is as follows: Facing the risk of a shock, the principal first chooses a technology that determines the quality of his private information about the risk of the shock. The technology is perfect if it enables the perfect forecasting of the shock, and imperfect otherwise. The set of forecasting technologies available for the principal equals $\boldsymbol{R}_{+} \cup\{\infty\}$, where 0 corresponds to the perfect technology and $\infty$ corresponds to the absence of the forecasting activity. The principal may costlessly choose any forecasting technology from this set. Upon acquiring information, the principal chooses whether to take his own preparatory action, and at the same time issues an advice to the agent on whether he should take a preparatory action. As mentioned above, these actions are costly to the respective parties, but their benefits accrue only when the shock strikes. The principal's payoffs are such that taking action is a dominant strategy for him in the event of a sure shock. That is, when the shock occurs for sure, the principal cannot commit to not taking action. As for the agent's payoffs in the shock state, on the other hand, we consider the following two possibilities. In the first case, taking action is also a dominant strategy for him. In the second case, taking action is a best response if and only if the principal does not. In the latter case, hence, the agent free-rides on the principal's effort.

Not surprisingly, we observe that when the agent has no free-riding incentive, acquiring perfect information and revealing it to the agent is the optimal policy for the principal. Our main focus hence is on the free-riding case. We first show that when the prior probability of the shock is moderately high, no information is better than the adoption of the perfect technology. When the prior probability is low, however, no information is dominated by perfect information. This leads us

\footnotetext{
${ }^{3}$ See Saito (2008).
} 
to the question on whether there still exists an imperfect information policy that outperforms perfect information even for low probability shocks. For this, we characterize an optimal disclosure rule and the associated equilibrium actions subject to imperfect information. Based on this characterization, we identify conditions under which there exists an information policy that dominates perfect information. The quality of information under such a policy is proportional to (some power of) the prior probability of the shock, and the action and advice are based on the three risk categories as follows: When the updated risk is high based on the acquired information, the principal takes action but recommends no action to the agent. When the risk is medium, the principal takes no action but recommends an action to the agent. When the risk is low, the principal takes no action and also recommends no action to the agent. We show that when the thresholds and information quality are appropriately chosen, such a policy dominates perfect information when the marginal benefit of the agent's action is sufficiently large for the principal, or when the agent's utility from inaction by both parties is sufficiently low in the event of the shock. These conditions are hence relevant when the public has significantly more efficient ways to insure against the risk, or when the shock has a disastrous consequence when no preparation is made.

We further proceed to the model in which the agent's free-riding incentive is also subject to uncertainty. We reinterpret the free-riding incentive as a measure of the severity of the shock, and suppose that when the shock is severe, the agent has no free-riding incentive. When the principal can acquire information about the probability and severity of a shock, we again observe that perfect information is dominated by a policy that acquires only imperfect information about the severity.

The paper is organized as follows: After the discussion of the related literature in the next section, we formulate in Section 3 a model of information acquisition and disclosure. Section 4 compares the two extreme cases of perfect information and complete obscurity. Section 5 describes an optimal disclosure rule subject to imperfect information. The main conclusion on the optimality of imperfect information is presented in Section 6. We conclude in Section 7.

\section{Related Literature}

The present paper is related to at least three different lines of research in the literature as follows. 
First, decision making in the face of information about a natural shock is a classical subject in both the theoretical and empirical literature. Nelson and Winter (1964) study the weather forecasting system that maximizes the welfare of a user of the forecast who must decide whether to take a protective action against rain. Howe and Cochrane (1974) study the decision problem faced by authorities under a snow storm forecast. While information acquisition is absent in their model, their empirical observation on the "reluctance on the part of snow removal authorities to be sensitive to any but very severe forecasts in making operation decisions" is consistent with the optimal policy in the current paper. Brookshire et al. (1985) show that the expected utility hypothesis is a reasonable description of decisionmaking behavior facing a low-probability, high-loss event of an earthquake. Lewis and Nickerson (1989) study the interaction of self-insurance and public interventions against natural disasters.

Second, the value of strategic ignorance is highlighted in dynamic contracting problems where the lack of commitment leads to renegotiation. Dewatripont and Maskin (1995) demonstrate the optimality of simple contracts that depend on the limited observation of variables. Cremer (1995) studies a dynamic principal-agent model with adverse selection and shows that the principal may refrain from acquiring information about the intrinsic productivity of an agent. Also in a principal-agent model with adverse selection, Kessler (1998) shows that an agent may optimally choose to remain ignorant about the state before contracting with a principal. ${ }^{4}$ Our model shares with these models the feature that the principal cannot commit to his action under perfect information. ${ }^{5}$ On the other hand, our main innovation is that we study the optimal degree of information imperfection and show that it can be a useful commitment device even when complete ignorance is not.

Third, information revelation and transparency are analyzed in various contexts. Among them, the present paper is related to the literature on the value of public information and government transparency. Beginning with the pioneering work of Hirschleifer (1971), the main theme of the literature is whether or not full disclosure of a government's private information induces an inefficient level of coordination

\footnotetext{
${ }^{4}$ Carrillo and Mariotti (2000) present another logic behind strategic ignorance by a decision maker with time-inconsistent preferences.

${ }^{5}$ Bester and Strausz (2000) study the possible failure of the revelation principle in such a problem. See also Skreta (2006). However, the problem of soliciting agents' private information is absent in our model since it is the principal who has private information in our model.
} 
by the public. ${ }^{6}$ While the literature is inconclusive as to whether transparency is socially optimal, the common assumption there is that a government's private information is exogenous. In contrast, we endogenize private information, and point to the possibility that it may be strictly less than what is potentially available.

\section{Model}

There are a principal (player 1) and an agent (player 2) facing the risk of a shock. The shock corresponds to one of the two states of the world $\omega \in \Omega$ : The shock occurs in state $\omega=1$ and does not in state $\omega=0$. The prior probability of the shock equals $p=P(\omega=1) \in(0,1)$. Before the state is realized, each player $i$ either "takes action" $\left(a_{i}=1\right)$ or not $\left(a_{i}=0\right)$ against the shock. We suppose that the principal and the agent take their actions simultaneously after the disclosure. ${ }^{7}$ The players' payoffs depend on the action profile and the state. Specifically, player $i$ 's payoff under the action profile $a=\left(a_{1}, a_{2}\right)$ in state $\omega$ is given by

$$
v_{i}(a, \omega)=u_{i}(a) \mathbf{1}_{\{\omega=1\}}-c_{i} \mathbf{1}_{\left\{a_{i}=1\right\}},
$$

where $\mathbf{1}_{A}$ denotes the indicator function of event $A$. Hence, the players benefit from the actions only when there is a shock $(\omega=1)$, but incur the cost $c_{i}$ of taking action even when there is no shock. Let

$$
\begin{array}{ll}
d_{1}^{0}=u_{1}(1,0)-u_{1}(0,0), & d_{1}^{1}=u_{1}(1,1)-u_{1}(0,1), \\
d_{2}^{0}=u_{2}(0,1)-u_{2}(0,0), & d_{2}^{1}=u_{2}(1,1)-u_{2}(1,0), \\
m_{1}^{0}=u_{1}(0,1)-u_{1}(0,0), & m_{1}^{1}=u_{1}(1,1)-u_{1}(1,0) .
\end{array}
$$

$d_{1}^{1}$ is the marginal benefit of his own action $a_{1}=1$ to the principal when the agent also chooses $a_{2}=1$, and $d_{1}^{0}$ is the marginal benefit of $a_{1}=1$ when it is unilaterally taken. $d_{2}^{0}$ and $d_{2}^{1}$ are the corresponding quantities for the agent. $m_{1}^{0}$ and $m_{1}^{1}$ are the marginal benefits of agent's action to the principal when the principal himself

\footnotetext{
${ }^{6}$ See, for example, Morris and Shin (2002) and Svensson (2006). Walsh (2007) analyzes the optimality of transparency when the quality of private information is varied.

${ }^{7}$ If the principal moves after the agent, it becomes more difficult to induce the agent to take action because the agent's inaction can more easily force the principal to take action. However, our conclusion holds true even with sequential moves if the agent is reinterpreted as a continuum of individuals none of whom can influence the principal's decision.
} 
chooses $a_{1}=0$ and $a_{1}=1$, respectively. We assume that

$$
\begin{aligned}
& d_{1}^{0} \geq d_{1}^{1}>c_{1}>0, \\
& d_{2}^{0}>d_{2}^{1}>0, \\
& m_{1}^{0}>d_{1}^{0}-c_{1} .
\end{aligned}
$$

(1) and (2) show that the two players' actions are strategic substitutes: The marginal benefit of the own action is higher when it is unilateral. (1) also says that $a_{1}=1$ is a dominant action for the principal in the event of a sure shock. We study two different possibilities concerning the magnitude of $d_{2}^{0}$ and $d_{2}^{1}$ relative to $c_{2}$. The agent has a free-riding incentive if

$$
d_{2}^{1}<c_{2}<d_{2}^{0}
$$

and has no free-riding incentive if $d_{2}^{1} \geq c_{2}$. (3) says that for the principal, the marginal benefit of the agent's unilateral action is higher than the net marginal benefit of his own unilateral action. For concreteness, we also assume in what follows that

$$
\frac{d_{2}^{0}}{c_{2}}>\frac{d_{1}^{0}}{c_{1}}
$$

In other words, when normalized by its cost, the agent's (unilateral) action raises his own utility more effectively than the principal's (unilateral) action raises his own utility.

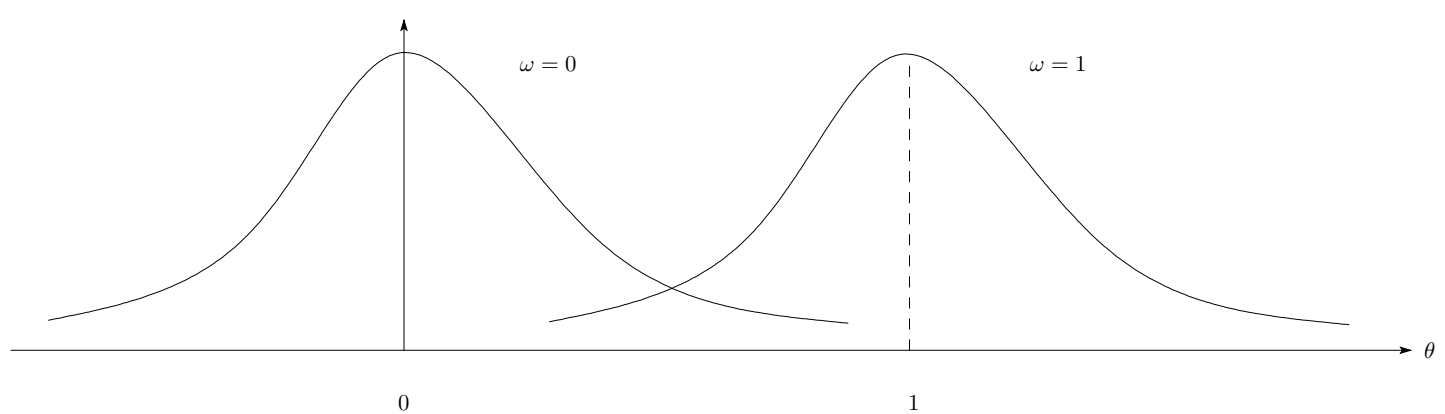

Figure 1: The signal distributions in states $\omega=0$ and $\omega=1$

The timing of the events is as follows. First, the principal chooses the forecasting technology $r$ that determines the precision of his private information $\theta$. We suppose that under the technology $r \geq 0$, the principal observes signal $\theta$ given by

$$
\theta=\mathbf{1}_{\{\omega=1\}}+r \epsilon
$$


where $\epsilon$ is a random noise term independent of $\omega$ and having positive density $f$ over $\boldsymbol{R}$. As seen in Figure 1, hence, the distribution of the signal $\theta$ shifts up in the state where the shock strikes. $r$ represents the level of noise, and we say that the technology $r$ is perfect if $r=0$ and imperfect otherwise. If we denote by $F$ the cumulative distribution of $\epsilon$, then the distribution $F_{\omega, r}$ of the signal $\theta$ under technology $r>0$ in state $\omega$ is expressed as

$$
F_{\omega, r}(\theta)=F\left(\frac{\theta-\mathbf{1}_{\{\omega=1\}}}{r}\right) .
$$

We assume that the corresponding density $f_{\omega, r}$ of $\theta$ satisfies the monotone likelihood ratio property as follows:

$$
x<y \quad \Rightarrow \quad \frac{f_{1, r}(x)}{f_{0, r}(x)}<\frac{f_{1, r}(y)}{f_{0, r}(y)}
$$

We also assume that $\lim _{x \rightarrow-\infty} \frac{f_{1, r}(x)}{f_{0, r}(x)}=0$ and $\lim _{x \rightarrow \infty} \frac{f_{1, r}(x)}{f_{0, r}(x)}=\infty$.

After observing $\theta$, the principal makes a non-binding advice to the agent on which action to take. Both parties then choose actions simultaneously. The principal's action choice is based on his signal $\theta$, while the agent's action choice is based on the principal's advice. Finally, the state is realized and the players receive payoffs.

The principal's choice of an advice given the observation of $\theta$ is expressed by a disclosure rule $g: \boldsymbol{R} \rightarrow A_{2}: g(\theta)$ is the action suggested to the agent when $\theta$ is observed. The principal's policy is a pair $(r, g)$ of his forecasting technology and disclosure rule. We do not impose any restriction on the functional form of the disclosure rule $g$. For example, it may advise action $a_{2}=1$ for $\theta$ above some threshold and $a_{2}=0$ below it, or $a_{2}=1$ for $\theta$ in some interval and $a_{2}=0$ otherwise. By the logic of the revelation principle, it entails no loss of generality to assume that the disclosure rule $g$ generates an advice to the agent and hence takes values in $A_{2} \cdot{ }^{8}$ We assume that the policy $(r, g)$ is chosen in advance and is publicly announced. Public observability of the forecasting technology $r$ is a reasonable assumption given that it usually entails publicly observable activities such as launching a satellite, building a supercomputer or a network of sensors, and so on. We also assume that the principal commits to his disclosure rule $g$ in the sense that for any signal $\theta$, his advice equals $g(\theta) .{ }^{9}$

\footnotetext{
${ }^{8}$ That is, even if $g$ reveals more information, any such information is associated with one of two actions $\theta$ by the agent. Hence, the principal can perform this conversion on behalf of the agent and advise on the action instead.

${ }^{9}$ This is a standard assumption in the information revelation literature, and is most likely justified
} 
Given a policy $(r, g)$, each player's strategy is defined as follows. The principal's strategy $\sigma_{1}: \boldsymbol{R} \rightarrow A_{1}$ chooses an action as a function of the observed signal $\theta$. On the other hand, the agent's strategy $\sigma_{2}: A_{2} \rightarrow A_{2}$ chooses an action as a function of the principal's advice. Let $\sigma_{2}^{*}$ denote the obedient strategy such that $\sigma_{2}^{*}\left(a_{2}\right)=a_{2}$ for any $a_{2} \in A_{2}$. Let $\pi_{i}(\sigma \mid r, g)$ denote player $i$ 's ex ante expected payoff under the strategy profile $\sigma=\left(\sigma_{1}, \sigma_{2}\right)$ and the policy $(r, g)$. Explicitly, they are given by

$$
\begin{aligned}
& \pi_{1}(\sigma \mid r, g)=E_{\omega, \theta}\left[u_{1}\left(\sigma_{1}(\theta), \sigma_{2}(g(\theta))\right) \mathbf{1}_{\{\omega=1\}}-c_{1} \mathbf{1}_{\left\{\sigma_{1}(\theta)=1\right\}}\right] \\
& \pi_{2}(\sigma \mid r, g)=E_{\omega, \theta}\left[u_{2}\left(\sigma_{1}(\theta), \sigma_{2}(g(\theta))\right) \mathbf{1}_{\{\omega=1\}}-c_{2} \mathbf{1}_{\left\{\sigma_{2}(g(\theta))=1\right\}}\right] .
\end{aligned}
$$

The strategy profile $\sigma$ is an equilibrium under $(r, g)$ if $\pi_{i}(\sigma \mid r, g) \geq \pi_{i}\left(\sigma_{i}^{\prime}, \sigma_{j} \mid r, g\right)$ for any $\sigma_{i}^{\prime}$ and $i \neq j$. A policy $(r, g)$ is incentive compatible if there exists a strategy $\sigma_{1}$ of the principal such that $\left(\sigma_{1}, \sigma_{2}^{*}\right)$ is an equilibrium under $(r, g)$.

A policy $(r, g)$ is optimal if it is incentive compatible and maximizes $\pi_{1}(\sigma \mid r, g)$ where $\sigma$ is an equilibrium under $(r, g)$. Formally, $(r, g)$ is optimal if there exists $\sigma_{1}$ such that $\sigma=\left(\sigma_{1}, \sigma_{2}^{*}\right)$ is an equilibrium under $(r, g)$, and for any policy $\left(r^{\prime}, g^{\prime}\right)$ and any equilibrium $\sigma^{\prime}$ under $\left(r^{\prime}, g^{\prime}\right)$, we have

$$
\pi_{1}(\sigma \mid r, g) \geq \pi_{1}\left(\sigma^{\prime} \mid r^{\prime}, g^{\prime}\right)
$$

\section{Perfect Information and Complete Obscurity}

We begin with the analysis of the principal's payoffs when he adopts the perfect technology $r=0$, and when he adopts the completely uninformative technology $r=\infty$.

First, in the case of the perfect technology, the principal observes either $\theta=0$ or $\theta=1$. It follows that the disclosure rule is either revealing $g(0) \neq g(1)$, or nonrevealing $g(0)=g(1)$. The following proposition makes a simple observation that if the agent knows that the principal knows the state, he knows that the principal takes action in state 1 . Hence, the free-riding agent does not take action in state 1 as a best response against the principal's taking action. This suggests that any incentive compatible disclosure rule must be non-revealing and advise no action whether $\theta=0$ or 1 .

for disclosure by a public sector, where adherence to the publicly announced rule is verifiable through official documents. 
Proposition 1 Suppose that $(r, g)$ is an incentive compatible policy with perfect information $r=0$ and admits an equilibrium $\sigma=\left(\sigma_{1}, \sigma_{2}^{*}\right)$.

1. If the agent has no free-riding incentive $\left(d_{2}^{1} \geq c_{2}\right)$, then

$$
\left(\sigma_{1}(\theta), g(\theta)\right)= \begin{cases}(1,1) & \text { if } \theta=1 \\ (0,0) & \text { otherwise }\end{cases}
$$

and

$$
\pi_{1}(\sigma \mid r, g)=p\left\{u_{1}(1,1)-c_{1}\right\}
$$

2. If the agent has a free-riding incentive $\left(d_{2}^{1}<c_{2}\right)$, then

$$
\left(\sigma_{1}(\theta), g(\theta)\right)= \begin{cases}(1,0) & \text { if } \theta=1, \\ (0,0) & \text { otherwise, }\end{cases}
$$

and the principal's ex ante expected equilibrium payoff equals

$$
\pi_{1}(\sigma \mid r, g)=p\left\{u_{1}(1,0)-c_{1}\right\} \equiv \pi_{1}^{0}
$$

Proof. Suppose that the agent has a free-riding incentive. Whether $g$ is revealing or not, the principal's equilibrium strategy $\sigma_{1}$ is as stated in the proposition. If $g$ is revealing $(g(0) \neq g(1))$, the agent chooses $a_{2}=0$ for any advice: When $\theta=1$, the unique NE is $\left(a_{1}, a_{2}\right)=(1,0)$ and when $\theta=0$, the unique NE is $(0,0)$. Hence, $(r, g)$ cannot be incentive compatible, and $g$ must be no-revealing. If $g$ is no-revealing, the agent chooses $a_{2}$ so as to maximize

$$
E\left[u_{2}\left(a_{1}=1, a_{2}\right) \mathbf{1}_{\{\theta=1\}}\right]-c_{2} \mathbf{1}_{\left\{a_{2}=1\right\}}=p u_{2}\left(1, a_{2}\right)-c_{2} \mathbf{1}_{\left\{a_{2}=1\right\}} .
$$

The maximizer is $a_{2}=0$ by assumption. Hence, the disclosure rule must be such that $g(\cdot)=0$ for $(r, g)$ to be incentive compatible. The principal's expected equilibrium payoff then equals $p\left\{u_{1}(1,0)-c_{1}\right\}$.

On the other hand, when the agent has no free-riding incentive, then acquiring perfect information does not prevent the agent from taking action, and the principal can recommend $a_{2}=1$ if and only if $\theta=1$.

Next, when the principal adopts the completely uninformative technology, we have the following observation. 
Proposition 2 Suppose that $(r, g)$ is an optimal incentive compatible policy with no information $r=\infty$, and admits an equilibrium $\sigma=\left(\sigma_{1}, \sigma_{2}^{*}\right)$.

1. If the agent has no free-riding incentive, then

$$
\left(\sigma_{1}, g\right)= \begin{cases}(0,0) & \text { if } p<\frac{c_{2}}{d_{2}^{0}} \\ (0,1) & \text { if } \frac{c_{2}}{d_{2}^{0}} \leq p<\frac{c_{1}}{d_{1}^{1}}, \\ (1,0) & \text { if } \frac{c_{1}}{d_{1}^{1}} \leq p<\max \left\{\frac{c_{1}}{d_{1}^{1}}, \frac{c_{2}}{d_{2}^{1}}\right\} \\ (1,1) & \text { if } p \geq \max \left\{\frac{c_{1}}{d_{1}^{1}}, \frac{c_{2}}{d_{2}^{1}}\right\}\end{cases}
$$

and

$$
\pi_{1}(\sigma \mid r, g)= \begin{cases}p u_{1}(0,0) & \text { if } p<\frac{c_{2}}{d_{2}^{0}} . \\ p u_{1}(0,1) & \text { if } \frac{c_{2}}{d_{2}^{0}} \leq p<\frac{c_{1}}{d_{1}^{1}} \\ p u_{1}(1,0)-c_{1} & \text { if } \frac{c_{1}}{d_{1}^{1}} \leq p<\max \left\{\frac{c_{1}}{d_{1}^{1}}, \frac{c_{2}}{d_{2}^{1}}\right\}, \\ p u_{1}(1,1)-c_{1} & \text { if } p \geq \max \left\{\frac{c_{1}}{d_{1}^{1}}, \frac{c_{2}}{d_{2}^{1}}\right\} .\end{cases}
$$

2. If the agent has a free-riding incentive (i.e., $\frac{c_{2}}{d_{2}^{1}}>1$ ), then

$$
\left(\sigma_{1}, g\right)= \begin{cases}(0,0) & \text { if } p<\frac{c_{2}}{d_{2}^{0}} \\ (0,1) & \text { if } \frac{c_{2}}{d_{2}^{0}} \leq p<\frac{c_{1}}{d_{1}^{1}} \\ (1,0) & \text { if } p \geq \frac{c_{1}}{d_{1}^{1}}\end{cases}
$$

and

$$
\pi_{1}(\sigma \mid r, g)= \begin{cases}p u_{1}(0,0) & \text { if } p<\frac{c_{2}}{d_{2}^{0}} . \\ p u_{1}(0,1) & \text { if } \frac{c_{2}}{d_{2}^{0}} \leq p<\frac{c_{1}}{d_{1}^{1}} \\ p u_{1}(1,0)-c_{1} & \text { if } p \geq \frac{c_{1}}{d_{1}^{1}}\end{cases}
$$

Proof. The principal's dominant action equals $a_{1}=1$ if $p>\frac{c_{1}}{d_{1}^{1}}$, and $a_{1}=0$ if $p<\frac{c_{1}}{d_{1}^{0}}$. When $\frac{c_{1}}{d_{1}^{0}} \leq p<\frac{c_{1}}{d_{1}^{1}}$, his best response is $a_{1}=1$ if $a_{2}=0$, and $a_{1}=0$ if $a_{2}=1$. On the other hand, the agent's best response against $a_{1}=0$ equals $a_{2}=1$ if $\frac{c_{2}}{d_{2}^{0}}<p<\frac{c_{1}}{d_{1}^{0}}$, and $a_{2}=0$ for $p<\frac{c_{2}}{d_{2}^{0}}$. If the agent has a free-riding incentive, hence, the action profile should be $a=(1,0)$ for $p>\frac{c_{1}}{d_{1}^{1}}$, and either $a=(1,0)$ or $(0,1)$ for $\frac{c_{1}}{d_{2}^{0}} \leq p<\frac{c_{1}}{d_{1}^{1}}$. By $(3)$, the optimal policy should have $a=(0,1)$ in this case. The disclosure rule $g$ is accordingly determined, and the principal's expected equilibrium 
payoff is as given in the proposition. The argument is similar when the agent has no free-riding incentive.

It immediately follows from Propositions 1 and 2 that against a free-riding agent, no information dominates perfect information when the prior probability of the shock is moderately high $\left(\frac{c_{2}}{d_{2}^{0}}<p<\frac{c_{1}}{d_{1}^{0}}\right)$ since $m_{1}>d_{1}^{0}-c_{1}$ by assumption and hence

$$
p u_{1}(0,1)>p\left\{u_{1}(1,0)-c_{1}\right\} .
$$

When the prior probability is low $\left(p<\frac{c_{2}}{d_{2}^{0}}\right)$, perfect information is better than no information since $d_{1}^{0}>c_{1}$ by assumption and hence

$$
p u_{1}(0,0)<p\left\{u_{1}(1,0)-c_{1}\right\}
$$

When the agent has no free-riding incentive, on the other hand, perfect information is not only better than no information but also is optimal among all policies given that the principal's payoff in state 1 cannot be improved further because $u_{1}(1,1)-$ $c_{1}>u_{1}(0,1)>u_{1}(1,0)-c_{1}>u_{1}(0,0)$.

The following corollary summarizes this observation.

Corollary 3 1. If the agent has no free-riding incentive, then perfect information policy $(r, g)$ as described in Proposition 1 is optimal among all policies.

2. If the agent has a free-riding incentive, then the following hold.

(a) If $p \leq \frac{c_{2}}{d_{2}^{0}}$ or $p>\frac{c_{1}}{d_{1}^{0}}$, then perfect information $(r=0)$ yields the higher expected payoff to the principal than no information $(r=\infty)$.

(b) If $p \in\left(\frac{c_{2}}{d_{2}^{0}}, \frac{c_{1}}{d_{1}^{0}}\right)$, then no information yields the higher expected payoff than perfect information.

We can interpret the observation in 2(b) above as one expression of the value of strategic ignorance as discussed in the literature mentioned in Section 2.

\section{Optimal Policy under Imperfect Information}

In this section, we fix an imperfect forecasting technology $r \in(0, \infty)$, and ask what type of disclosure rule $g$ makes $(r, g)$ both incentive compatible and optimal subject to the technology. This result is of interest in its own right when acquiring perfect information is technologically infeasible. The analysis is independent of whether the 
agent has a free-riding incentive or not. We use the characterization derived here to identify an imperfect policy that dominates perfect information in the next section.

Introduce the following notation:

$$
\begin{array}{ll}
\delta_{1}^{0}=\frac{d_{1}^{0}}{c_{1}}-1, & \delta_{1}^{1}=\frac{d_{1}^{1}}{c_{1}}-1, \\
\delta_{2}^{0}=\frac{d_{2}^{0}}{c_{2}}-1, & \delta_{2}^{1}=\frac{d_{2}^{1}}{c_{2}}-1, \\
\mu_{1}^{0}=\frac{m_{1}^{0}}{c_{1}}, & \mu_{1}^{1}=\frac{m_{1}^{1}}{c_{1}} .
\end{array}
$$

$\delta_{1}^{0}$ is the net marginal benefit for the principal of his own action normalized by its cost when the agent does not take action, and $\delta_{1}^{1}$ is the corresponding quantity when the agent takes action. $\delta_{2}^{0}$ and $\delta_{2}^{1}$ have similar interpretations. $\mu_{1}^{0}$ is the normalized marginal benefit for the principal of the agent's action. Let $\beta^{0}$ and $\beta^{1}$ be defined by the values of $\theta$ such that

$$
P\left(\omega=1 \mid \theta=\beta^{0}\right)=\frac{c_{1}}{d_{1}^{0}}, \quad \text { and } \quad P\left(\omega=1 \mid \theta=\beta^{1}\right)=\frac{c_{1}}{d_{1}^{1}} .
$$

These are equivalent to

$$
\frac{f_{0, r}\left(\beta^{0}\right)}{f_{1, r}\left(\beta^{0}\right)}=\frac{p \delta_{1}^{0}}{1-p} \quad \text { and } \quad \frac{f_{0, r}\left(\beta^{1}\right)}{f_{1, r}\left(\beta^{1}\right)}=\frac{p \delta_{1}^{1}}{1-p} .
$$

Since by assumption, $\frac{f_{0, r}(\theta)}{f_{1, r}(\theta)}$ strictly decreases from $\infty$ to 0 as $\theta$ varies from $-\infty$ to $\infty, \beta^{0}$ and $\beta^{1}$ are well-defined and unique, and satisfy $\beta^{0}<\beta^{1}$ since $\delta_{1}^{1}<\delta_{1}^{0}$. It can be verified that $\beta^{0}$ is the signal value at which the principal is exactly indifferent between taking action and not when the agent does not take action. Likewise, $\beta^{1}$ is the signal value at which the principal is indifferent when the agent takes action:

$$
\begin{aligned}
& \theta=\beta^{0} \quad \Leftrightarrow \quad E_{\omega}\left[u_{1}(1,0) \mathbf{1}_{\{\omega=1\}}-c_{1} \mid \theta\right]=E_{\omega}\left[u_{1}(0,0) 1_{\{\omega=1\}} \mid \theta\right], \\
& \theta=\beta^{1} \quad \Leftrightarrow \quad E_{\omega}\left[u_{1}(1,1) 1_{\{\omega=1\}}-c_{1} \mid \theta\right]=E_{\omega}\left[u_{1}(0,1) 1_{\{\omega=1\}} \mid \theta\right] .
\end{aligned}
$$

It follows that the principal always takes action when $\theta>\beta^{1}$, and that he never takes action when $\theta<\beta^{0}$. The following proposition characterizes an optimal disclosure rule $g$ and the associated equilibrium $\sigma$ given $r>0$.

Proposition 4 Suppose that $(r, g)$ is an optimal incentive compatible policy with $r \in(0, \infty)$ and admits an equilibrium $\sigma=\left(\sigma_{1}, \sigma_{2}^{*}\right)$. Then there exist $\alpha, \nu$ and $\gamma$ 
such that $\alpha \leq \beta^{0} \leq \nu \leq \beta^{1} \leq \gamma \leq \infty$ and

$$
\left(\sigma_{1}(\theta), g(\theta)\right)= \begin{cases}(0,0) & \text { for almost every } \theta \in(-\infty, \alpha), \\ (0,1) & \text { for almost every } \theta \in\left[\alpha, \beta^{0}\right) \cup\left[\nu, \beta^{1}\right), \\ (1,0) & \text { for almost every } \theta \in\left[\beta^{0}, \nu\right) \cup\left[\beta^{1}, \gamma\right), \\ (1,1) & \text { for almost every } \theta \in[\gamma, \infty) .\end{cases}
$$

Proposition 4 is illustrated in Figure 2. In each one of the three intervals $\left(-\infty, \beta^{0}\right),\left(\beta^{0}, \beta^{1}\right)$, and $\left(\beta^{1}, \infty\right)$, the optimal disclosure rule advises the agent to take action when the signal is in the upper portion of the interval. The proposition also suggests that the problem of finding an optimal disclosure rule can be reduced to the problem of finding the optimal thresholds $\alpha, \nu$ and $\gamma$.

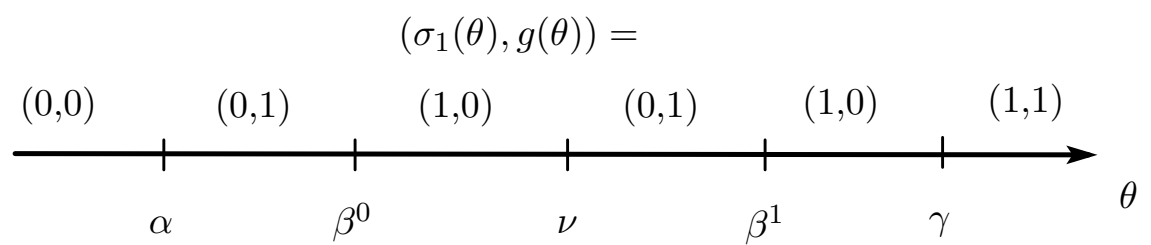

Figure 2: Optimal disclosure rule under imperfect information

\section{Optimality of Obscurity for a Free-Riding Agent}

Given the observation in Corollary 3 that against a free-riding agent, no information dominates perfect information when the prior probability of a shock is moderately high $p \in\left(\frac{c_{2}}{d_{2}^{0}}, \frac{c_{1}}{d_{1}^{0}}\right)$, we now focus on the case where it is low:

$$
p \in\left(0, \frac{c_{2}}{d_{2}^{0}}\right]
$$

For such a $p$, we will identify conditions on the payoffs under which there exists a policy $(r, g)$ with $r>0$ that dominates perfect information.

For concreteness, we assume in what follows that the noise term in $\theta$ has the standard normal distribution $N(0,1)$ over $\boldsymbol{R}$. It follows that when the principal adopts technology $r>0, \theta$ has the normal distribution with mean $\mu=1_{\{\omega=1\}}$ and variance $r^{2}$ in state $\omega$. 
Theorem 5 Suppose that the agent has a free-riding incentive. Suppose further that $\mu_{1}^{0}-\delta_{1}^{0}-\delta_{1}^{1}>0$ and that

$$
\frac{\mu_{1}^{0}}{\mu_{1}^{0}-\delta_{1}^{0}-\delta_{1}^{1}}<\left(\frac{\delta_{2}^{0}}{\delta_{1}^{1}}\right)^{\frac{1}{2}} .
$$

Then for any prior probability $p \in\left(0, \frac{c_{2}}{d_{2}^{0}}\right)$, there exists $r>0$ such that for $\left(\sigma_{1}, g\right)$ given below, $(r, g)$ is incentive compatible, admits an equilibrium $\sigma=\left(\sigma_{1}, \sigma_{2}^{*}\right)$, and yields a strictly higher payoff to the principal than perfect information.

$$
\sigma_{1}(\theta)=\left\{\begin{array}{ll}
1 & \text { if } \theta \geq \beta, \\
0 & \text { otherwise, }
\end{array} \quad g(\theta)= \begin{cases}1 & \text { if } \theta \in[\alpha, \beta), \\
0 & \text { otherwise }\end{cases}\right.
$$

where $\alpha$ is given by $P(\omega=1 \mid \theta=\alpha)=\frac{c_{2}}{d_{2}^{0}}$, or equivalently,

$$
\frac{f_{0}(\alpha)}{f_{1}(\alpha)}=\frac{p \delta_{2}^{0}}{1-p}
$$

and $\beta$ equals $\beta^{1}$ defined in (6).

According to the policy described in the theorem, the principal takes action himself and advises no action to the agent when taking action is a dominant strategy for him, and takes no action and advises the agent to take action when $a=(0,1)$ is an equilibrium action. This disclosure rule belongs to the class identified in Proposition $4{ }^{10}$ We can also see that this disclosure rule is equivalent to the full revelation of $\theta$ as follows: If the agent also observes $\theta, a=(0,1)$ is an equilibrium action profile when the posterior probability $P(\omega=1 \mid \theta) \in\left[\frac{c_{2}}{d_{2}^{0}}, \frac{c_{1}}{d_{1}^{1}}\right)$. Likewise, $a=(1,0)$ is an equilibrium when $P(\omega=1 \mid \theta)>\frac{c_{1}}{d_{1}^{1}}$, and $a=(0,0)$ is an equilibrium when $P(\omega=1 \mid \theta)<\frac{c_{2}}{d_{2}^{0}}$. In other words, this policy is transparent in the communication stage although it entails imperfection at the information acquisition stage.

Clearly, the cost of imperfect information for the principal is the cost of wasted effort in state 0 . On the other hand, the benefit is that he can commit to no action and have the agent take action when the risk is medium and $a=(0,1)$ is an equilibrium. The point is whether the probability of observing such a signal is made sufficiently large compared with the probability of observing a high signal in state 0 . The above proposition shows that this is possible when the signal quality is appropriately chosen provided that the payoffs satisfy the stated conditions.

\footnotetext{
${ }^{10}$ Specifically, set $\gamma=\infty$ and $\nu=\beta^{0}$ in Proposition 4.
} 
The sufficient conditions in Theorem 5 are strictly in terms of the payoffs and independent of the prior probability $p$. The condition (9) clearly holds when $\delta_{2}^{0}$ is sufficiently large so that the agent has a strong incentive to make effort in the absence of the principal's effort. Alternatively, it holds when the agent's action has a significant positive impact on the principal's payoff. For example, suppose that the principal's payoff is written in the form $u_{1}(a)=\left(d_{2}^{0}-a_{2}\right) a_{1}+m_{1}^{0} a_{2}$, where $m_{1}^{0}=u_{1}(0,1)-u_{1}(0,0)$ is the benefit of the agent's action for the principal. As $m_{1}^{0}$ increases, so does $\mu_{1}^{0}=\frac{m_{1}^{0}}{c_{1}}$, while $\delta_{1}^{0}$ and $\delta_{1}^{1}$ remain unchanged. Thus, the lefthand side of (9) decreases, and the conditions of the theorem will eventually hold. These qualitative interpretations of (9) are in line with the cost-benefit analysis of imperfect information described above: The range $\left(\frac{c_{2}}{d_{2}^{0}}, \frac{c_{1}}{d_{1}^{1}}\right)$ of posterior probabilities is where the principal takes no action and lets the agent take action. Hence, the principal is better off if $d_{2}^{0}$ is larger so that this interval is larger, or when he receives a significant benefit from the agent's unilateral action.

For illustration, Figure 3 depicts the difference $\pi_{1}(\sigma \mid r, g)-\pi_{1}^{0}$ divided by the prior probability $p$ of a shock as a function of $p$ when the payoff numbers are given by $^{11}$

$$
\begin{aligned}
& u_{1}(1,1)=212, u_{1}(0,1)=201, u_{1}(1,0)=12, u_{1}(0,0)=0, \\
& u_{2}(0,1)=29, u_{2}(0,0)=0, \text { and } c_{1}=c_{2}=1 .
\end{aligned}
$$

\section{Information about the Severity of a Shock}

In many circumstances, the severity of a shock is also a primary object of a forecast along with its probability of occurrence. We study in this section what happens when the principal can acquire and disclose information about both the probability and severity of a shock. Among different ways of modeling severity, we define in what follows that the shock is severe if the agent has no free-riding incentive, and not severe otherwise. The interpretation is that when the shock is severe, the agent cannot simply rely on the principal to mitigate the damage. Given that the conclusions in the preceding sections depend on whether the agent has a free-riding

\footnotetext{
${ }^{11}$ Note that perfect information yields $\frac{\pi_{1}^{0}}{p}=u_{1}(1,0)-c_{1}=11$, and that $u_{2}(1,1)$ and $u_{2}(1,0)$ are not relevant for the computation of $\pi_{1}(\sigma \mid r, g)$. We have $\delta_{1}^{0}=11, \delta_{1}^{1}=10, \mu_{1}^{0}=201$, and $\delta_{2}^{0}=28$, and the conditions of the theorem are satisfied since $\mu_{1}^{0}-\delta_{1}^{0}-\delta_{1}^{1}=180>0$, and $\frac{\mu_{1}^{0}}{\mu_{1}^{0}-\delta_{1}^{0}-\delta_{1}^{1}}=\frac{67}{60}<\left(\frac{\delta_{2}^{0}}{\delta_{1}^{1}}\right)^{\frac{1}{2}}=\left(\frac{7}{5}\right)^{\frac{1}{2}}$. The upper bound of $p$ as indicated in (8) equals $\frac{c_{2}}{d_{2}^{0}}=\frac{1}{29} \fallingdotseq 0.034$, which corresponds to the right-end of the horizontal axis in Figure 3.
} 


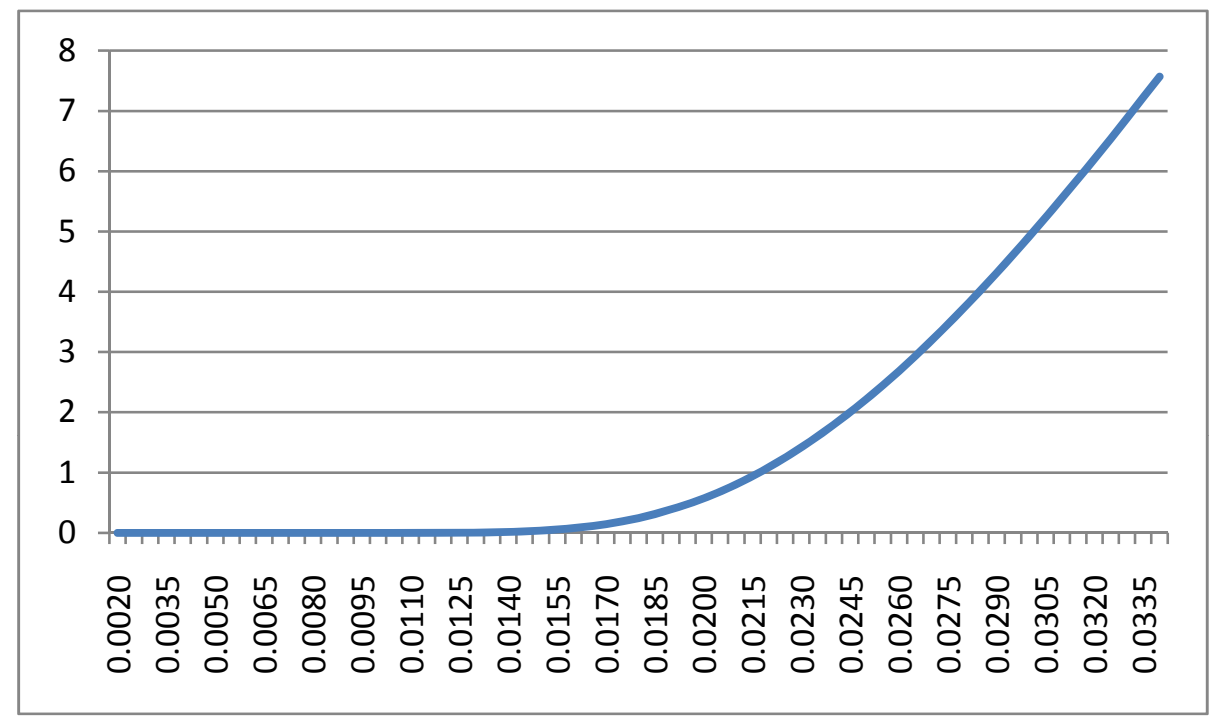

Figure 3: $\frac{1}{p}\left(\pi_{1}(\sigma \mid r, g)-\pi_{1}^{0}\right)$ plotted against $p$

incentive, we think that introducing uncertainty about this incentive provides an interesting exercise.

Formally, suppose that the state is now given by a vector $\omega=\left(\omega_{p}, \omega_{q}\right) \in\{0,1\}^{2}$, where $\omega_{p}$ denotes whether a shock occurs and $\omega_{q}$ denotes whether it is severe. That is, $\omega_{p}=1$ if the shock occurs and $\omega_{q}=1$ if it is severe. ${ }^{12}$ Let $q \in(0,1)$ denote the probability that the shock is severe conditional on its occurrence. Hence, we have $P(\omega=(1,0))=p(1-q)$ and $P(\omega=(1,1))=p q$. For simplicity, we assume that only the agent's utility function $u_{2}$ depends on $\omega_{q}$, and satisfies

$$
d_{2}^{0}\left(\omega_{q}\right) \equiv u_{2}\left(0,1, \omega_{q}\right)-u_{2}\left(0,0, \omega_{q}\right)>c_{2} \text { for } \omega_{q}=0,1,
$$

and

$$
d_{2}^{1}\left(\omega_{q}\right) \equiv u_{2}\left(1,1, \omega_{q}\right)-u_{2}\left(1,0, \omega_{q}\right) \begin{cases}>c_{2} & \text { for } \omega_{q}=1, \\ <c_{2} & \text { for } \omega_{q}=0 .\end{cases}
$$

In other words, when the shock is certain and is severe (i.e, in state $\omega=(1,1)$ ), the agent's best response against $a_{1}=1$ is to take action. When it is not severe, his best response against $a_{1}=1$ is to take no action.

Suppose now that the principal can acquire information about both $\omega_{p}$ and $\omega_{q}$. When we denote by $r_{p}$ and $r_{q}$ the principal's technologies used to forecast $\omega_{p}$ and

\footnotetext{
${ }^{12}$ The two states $\omega=(0,0)$ and $(0,1)$ are identified since no shock occurs in these states.
} 
$\omega_{q}$, respectively, his private signal $\theta$ is given by

$$
\theta_{p}=\mathbf{1}_{\left\{\omega_{p}=1\right\}}+r_{p} \epsilon_{p}, \text { and } \theta_{q}=\mathbf{1}_{\left\{\omega_{q}=1\right\}}+r_{q} \epsilon_{q},
$$

where $\epsilon_{p}$ and $\epsilon_{q}$ are independent random variables having the standard normal distribution $N(0,1)$. The distribution of $\theta_{p}$ shifts up in the state where the shock occurs and that of $\theta_{q}$ shifts up in the state where it is severe. A disclosure rule $g$ is a mapping from $\boldsymbol{R}^{2}$ to $A_{2}$, so that $g\left(\theta_{p}, \theta_{q}\right)$ is an advice to the agent when the signal pair is $\theta=\left(\theta_{p}, \theta_{q}\right)$. We suppose that $r_{p}$ and $r_{q}$ are chosen simultaneously, and are interested in determining whether the principal is better off acquiring imperfect information about either $\omega_{p}$ or $\omega_{q}\left(r=\left(r_{p}, r_{q}\right) \neq 0\right)$ than acquiring perfect information about both $(r=0)$.

Consider first the principal's expected payoff under perfect information $r=0$. In this case, it is readily seen that the optimal incentive compatible policy $(r, g)$, the equilibrium strategy $\sigma=\left(\sigma_{1}, \sigma_{2}^{*}\right)$, and the corresponding expected payoff of the principal are given as follows:

1. When $q \geq \frac{c_{2}-\delta_{2}^{1}(0)}{\delta_{2}^{1}(1)-\delta_{2}^{1}(0)}$,

$$
\left(\sigma_{1}(\theta), g(\theta)\right)= \begin{cases}(1,1) & \text { if } \theta=(1,1) \text { or }(1,0), \\ (0,0) & \text { otherwise }\end{cases}
$$

and $\pi_{1}(\sigma \mid r, g)=p\left\{u_{1}(1,1)-c_{1}\right\}$.

2. When $q<\frac{c_{2}-\delta_{2}^{1}(0)}{\delta_{2}^{1}(1)-\delta_{2}^{1}(0)}$,

$$
\left(\sigma_{1}(\theta), g(\theta)\right)= \begin{cases}(1,1) & \text { if } \theta=(1,1) \\ (1,0) & \text { if } \theta=(1,0) \\ (0,0) & \text { otherwise }\end{cases}
$$

and $\pi_{1}(\sigma \mid r, g)=p\left\{q u_{1}(1,1)+(1-q) u_{1}(1,0)-c_{1}\right\}=\pi_{1}^{0} \cdot{ }^{13}$

Note that when the ex ante probability of a severe shock is high, it is optimal for the principal to reveal no information about $\omega_{q}$ even if he observes it. Since acquisition of no information about $\omega_{q}$ yields the same result in this case, we will focus on the case where the probability $q$ is low.

\footnotetext{
${ }^{13}$ We don't consider a random policy that generates advice $a_{2}=1$ with positive probability when $\theta_{q}=0$ to make the agent just indifferent between taking action and not. We think that such a policy lacks credibility and is unrealistic.
} 
Proposition 6 Suppose that $q<\frac{c_{2}-\delta_{2}^{1}(0)}{\delta_{2}^{1}(1)-\delta_{2}^{1}(0)}$. Then there exists an imperfect information policy $(r, g)$ with $r_{q}>0$ that is incentive compatible and yields a higher expected equilibrium payoff to the principal than perfect information $r=\left(r_{p}, r_{q}\right)=0$.

We again observe the optimality of imperfect information. The policy $(r, g)$ that dominates perfect information in this case has $r_{p}=0$ and $r_{q}>0$, and advises $a_{2}=1$ when $\theta_{q} \geq \alpha$ for some threshold $\alpha$. Since $r_{p}=0$, this policy entails no wasted effort on the part of the principal. The principal in this model wants to convince the agent that $\omega_{q}=1$ in both states. When the technology $r_{q}$ and threshold $\alpha$ are appropriately chosen, we can show that the probability $P\left(\theta_{q} \geq \alpha \mid \omega_{q}=0\right)$ that the agent takes action in state 0 is made sufficiently large compared with the probability $P\left(\theta_{q}<\alpha \mid \omega_{q}=1\right)$ that the agent takes no action in state 1 . We are yet to see if the optimal policy has imperfect information about both $\omega_{p}$ and $\omega_{q}$.

\section{Conclusion}

In a model of information acquisition and disclosure, we show that endogenous information about the risk of a shock may be imperfect when the agent may free ride on the principal's preparation efforts. For a shock with moderately high prior probability, the principal prefers no information to perfect information. On the other hand, for a shock with small prior probability, the principal prefers perfect information to no information, but the optimal policy may entail a strictly positive degree of imperfection. Specifically, we show that the full revelation of imperfect information may outperform perfect information.

The model adopts an extreme assumption that a perfectly informative signal is costlessly available to the principal. Of course, positive cost of information acquisition only reinforces the main conclusion of the paper. When acquisition of perfect information is technologically infeasible, the relevant question is whether the optimal information is less precise than what is technologically feasible. The answer naturally depends on the parameters, but the basic intuition of the present analysis continues to be valid.

The scientific assessment of a risk is often very difficult to communicate to nonexperts. Furthermore, it is often observed that individuals overreact to a small probability risk in some cases, and undermine a moderately high probability risk in other cases. In this sense, the biggest challenge for the sender of information may 
be to induce the right action from the receivers taking into account the imperfection and bias in their information processing. ${ }^{14}$ Theoretical investigation into such a process would be an interesting topic of future research.

\section{Appendix}

Proof of Proposition 4 Since $\sigma=\left(\sigma_{1}, \sigma_{2}^{*}\right)$ is an equilibrium under $(r, g)$, we have $\sigma_{1}(\theta)=1$ if

$$
E_{\omega}\left[u_{1}(1, g(\theta)) \mathbf{1}_{\{\omega=1\}} \mid \theta\right]-c_{1}>E_{\omega}\left[u_{1}(0, g(\theta)) \mathbf{1}_{\{\omega=1\}} \mid \theta\right],
$$

or equivalently

$$
\left\{u_{1}(1, g(\theta))-u_{1}(0, g(\theta))\right\} P(\omega=1 \mid \theta)>c_{1} .
$$

Since $d_{1}^{0}>d_{1}^{1}$ by assumption, $\sigma_{1}(\theta)=1$ must hold if

$$
P(\omega=1 \mid \theta)=\frac{p f_{1}(\theta)}{p f_{1}(\theta)+(1-p) f_{0}(\theta)}>\frac{c_{1}}{d_{1}^{1}},
$$

which is equivalent to $\theta>\beta^{1}$. Likewise, we have $\sigma_{1}(\theta)=0$ if $\theta<\beta^{0}$. We can also conclude from these that

$$
\begin{gathered}
\theta>\beta^{1} \quad \Rightarrow \quad\left(\sigma_{1}(\theta), g(\theta)\right) \in\{(1,0),(1,1)\} \\
\theta \in\left(\beta^{0}, \beta^{1}\right) \quad \Rightarrow \quad\left(\sigma_{1}(\theta), g(\theta)\right) \in\{(1,0),(0,1)\} \\
\theta<\beta^{0} \Rightarrow \quad\left(\sigma_{1}(\theta), g(\theta)\right) \in\{(0,0),(0,1)\}
\end{gathered}
$$

We next show that there exists $\nu \in\left[\beta^{0}, \beta^{1}\right]$ such that $g(\theta)=0$ for almost every $\theta \in\left(\beta^{0}, \nu\right)$ and $g(\theta)=1$ for almost every $\theta \in\left(\nu, \beta^{1}\right)$. The proofs for the existence of $\alpha$ and $\gamma$ as stated in the theorem are almost identical and omitted.

Fix $(r, g)$ such that $r>0$. Let

$$
B_{0}=\{\theta \in \boldsymbol{R}: g(\theta)=0\}, \text { and } B_{1}=\{\theta \in \boldsymbol{R}: g(\theta)=1\} .
$$

We claim that if $g$ is optimal, then for any $x \in\left(\beta^{0}, \beta^{1}\right)$,

$$
P\left(\theta \in\left(\beta^{0}, x\right) \cap B_{1}\right)>0 \Rightarrow P\left(\theta \in\left(x, \beta^{1}\right) \cap B_{0}\right)=0 .
$$

Intuitively, (12) states that when $g$ advises $a_{2}=1$ for some signal $\theta$, then it should advise $a_{2}=1$ for almost every signal above $\theta$.

\footnotetext{
${ }^{14}$ See, for example, Eggers and Fischhoff (2004) and Fischhoff $(1994,2011)$ for the discussion of communication strategies when the receivers have limited capabilities.
} 
Suppose to the contrary that $P\left(\theta \in\left(\beta^{0}, x\right) \cap B_{1}\right)>0$ but $P\left(\theta \in\left(x, \beta^{1}\right) \cap B_{0}\right)>0$ for some such $x$. We will show that $(r, g)$ is dominated by an alternative policy. Take $D_{1} \subset\left(\beta^{0}, x\right) \cap B_{1}$ and $D_{0} \subset\left(x, \beta^{1}\right) \cap B_{0}$ such that

$$
P\left(\theta \in D_{0} \mid \omega=0\right)=P\left(\theta \in D_{1} \mid \omega=0\right)>0 .
$$

Define $\hat{B}_{1}$ and $\hat{B}_{0}$ by

$$
\begin{aligned}
& \hat{B}_{1} \equiv B_{1} \cup D_{0} \backslash D_{1}, \\
& \hat{B}_{0} \equiv B_{0} \cup D_{1} \backslash D_{0},
\end{aligned}
$$

and $\hat{\sigma}=\left(\hat{\sigma}_{1}, \sigma_{2}^{*}\right)$ and $\hat{g}$ by

$$
\left(\hat{g}(\theta), \hat{\sigma}_{1}(\theta)\right)= \begin{cases}(1,0) & \text { if } \theta \in D_{0} \\ (0,1) & \text { if } \theta \in D_{1} \\ \left(g(\theta), \sigma_{1}(\theta)\right) & \text { otherwise }\end{cases}
$$

In other words, $\left(\sigma_{1}, g\right)$ and $\left(\hat{\sigma}_{1}, \hat{g}\right)$ are switched on the sets $D_{0}$ and $D_{1}$, but are the same otherwise.

Since every element of $D_{0}$ is larger than any element of $D_{1}$, the monotone likelihood ratio property implies that

$$
\frac{P\left(\theta \in D_{0} \mid \omega=1\right)}{P\left(\theta \in D_{0} \mid \omega=0\right)}>\frac{P\left(\theta \in D_{1} \mid \omega=1\right)}{P\left(\theta \in D_{1} \mid \omega=0\right)} .
$$

We hence have

$$
P\left(\theta \in D_{0} \mid \omega=1\right)>P\left(\theta \in D_{1} \mid \omega=1\right) .
$$

We now claim that

$$
\begin{aligned}
& P\left(\theta<\beta^{1}, \omega=1 \mid \theta \in \hat{B}_{1}\right)>P\left(\theta<\beta^{1}, \omega=1 \mid \theta \in B_{1}\right), \\
& P\left(\omega=1 \mid \theta \in \hat{B}_{1}\right)>P\left(\omega=1 \mid \theta \in B_{1}\right), \\
& P\left(\theta>\beta^{1}, \omega=1 \mid \theta \in \hat{B}_{1}\right)<P\left(\theta>\beta^{1}, \omega=1 \mid \theta \in B_{1}\right), \\
& P\left(\omega=1 \mid \theta \in \hat{B}_{1}\right)<P\left(\theta>\beta^{1}, \omega=1 \mid \theta \in B_{1}\right) .
\end{aligned}
$$


The first inequality in (14) holds because

$$
\begin{aligned}
& P\left(\theta<\beta^{1}, \omega=1 \mid \theta \in \hat{B}_{1}\right) \\
& =\frac{p P\left(\theta \in\left(-\infty, \beta^{1}\right) \cap \hat{B}_{1} \mid \omega=1\right)}{P\left(\theta \in \hat{B}_{1}\right)} \\
& =\frac{p P\left(\theta \in\left(-\infty, \beta^{1}\right) \cap\left(B_{1} \cup D_{0} \backslash D_{1}\right) \mid \omega=1\right)}{P\left(\theta \in B_{1} \cup D_{0} \backslash D_{1}\right)} \\
& =\frac{p\left\{P\left(\theta \in\left(-\infty, \beta^{1}\right) \cap B_{1} \mid \omega=1\right)+P\left(\theta \in D_{0} \mid \omega=1\right)-P\left(\theta \in D_{1} \mid \omega=1\right)\right\}}{P\left(\theta \in B_{1}\right)+P\left(\theta \in D_{0}\right)-P\left(\theta \in D_{1}\right)} \\
& =\frac{p P\left(\theta \in\left(-\infty, \beta^{1}\right) \cap B_{1} \mid \omega=1\right)+p\left\{P\left(\theta \in D_{0} \mid \omega=1\right)-P\left(\theta \in D_{1} \mid \omega=1\right)\right\}}{P\left(\theta \in B_{1}\right)+p\left\{P\left(\theta \in D_{0} \mid \omega=1\right)-P\left(\theta \in D_{1} \mid \omega=1\right)\right\}} \\
& >P\left(\theta<\beta^{1}, \omega=1 \mid \theta \in B_{1}\right),
\end{aligned}
$$

where the last equality follows from (13). The derivation of the other inequalities in (14) is similar. Together, these inequalities show that if $(r, g)$ is incentive compatible, then so is $(r, \hat{g})$. To see this, note that $(r, g)$ is incentive compatible if

$$
\begin{aligned}
& d_{2}^{0} P\left(\theta<\beta^{1}, \omega=1 \mid \theta \in B_{1}\right)+d_{2}^{1} P\left(\theta>\beta^{1}, \omega=1 \mid \theta \in B_{1}\right) \geq c_{2}, \\
& d_{2}^{0} P\left(\theta<\beta^{1}, \omega=1 \mid \theta \in B_{0}\right)+d_{2}^{1} P\left(\theta>\beta^{1}, \omega=1 \mid \theta \in B_{0}\right) \leq c_{2},
\end{aligned}
$$

and that $(r, \hat{g})$ is incentive compatible if

$$
\begin{aligned}
& d_{2}^{0} P\left(\theta<\beta^{1}, \omega=1 \mid \theta \in \hat{B}_{1}\right)+d_{2}^{1} P\left(\theta>\beta^{1}, \omega=1 \mid \theta \in \hat{B}_{1}\right) \geq c_{2}, \\
& d_{2}^{0} P\left(\theta<\beta^{1}, \omega=1 \mid \theta \in \hat{B}_{0}\right)+d_{2}^{1} P\left(\theta>\beta^{1}, \omega=1 \mid \theta \in \hat{B}_{0}\right) \leq c_{2} .
\end{aligned}
$$

If (14) holds, then

$$
\begin{aligned}
& P\left(\theta<\beta^{1}, \omega=1 \mid \theta \in \hat{B}_{1}\right)-P\left(\theta<\beta^{1}, \omega=1 \mid \theta \in B_{1}\right) \\
& +P\left(\theta>\beta^{1}, \omega=1 \mid \theta \in \hat{B}_{1}\right)-P\left(\theta>\beta^{1}, \omega=1 \mid \theta \in B_{1}\right) \\
& =P\left(\omega=1 \mid \theta \in \hat{B}_{1}\right)-P\left(\omega=1 \mid \theta \in B_{1}\right)>0
\end{aligned}
$$

Hence,

$$
\begin{aligned}
& d_{2}^{0}\left\{P\left(\theta<\beta^{1}, \omega=1 \mid \theta \in \hat{B}_{1}\right)-P\left(\theta<\beta^{1}, \omega=1 \mid \theta \in B_{1}\right)\right\} \\
& +d_{2}^{1}\left\{P\left(\theta>\beta^{1}, \omega=1 \mid \theta \in \hat{B}_{1}\right)-P\left(\theta>\beta^{1}, \omega=1 \mid \theta \in B_{1}\right)\right\} \\
& >\left(d_{2}^{0}-d_{2}^{1}\right)\left\{P\left(\theta<\beta^{1}, \omega=1 \mid \theta \in \hat{B}_{1}\right)-P\left(\theta<\beta^{1}, \omega=1 \mid \theta \in B_{1}\right)\right\} \\
& >0 .
\end{aligned}
$$


It follows that the first condition for the incentive compatibility of $(r, g)$ implies that of $(r, \hat{g})$. Likewise, the second condition for the incentive compatibility of $(r, g)$ implies that of $(r, \hat{g})$ under (14).

Note now that the principal's payoff under $(r, g)$ and $\sigma$ can be written as

$$
\begin{aligned}
\pi_{1}(\sigma \mid r, g)= & p\left[\left\{u_{1}(1,0)-c_{1}\right\} P\left(\theta \in\left(\beta^{0}, \beta^{1}\right) \cap B_{0} \mid \omega=1\right)\right. \\
& \left.+u_{1}(0,1) P\left(\theta \in\left(\beta^{0}, \beta^{1}\right) \cap B_{1} \mid \omega=1\right)\right] \\
& -(1-p) c_{1} P\left(\theta \in\left(\beta^{0}, \beta^{1}\right) \cap B_{0} \mid \omega=0\right) \\
& +E_{\omega}\left[\left\{u_{1}\left(\sigma_{1}(\theta), g(\theta)\right) \mathbf{1}_{\{\omega=1\}}-c_{1} \mathbf{1}_{\left\{\sigma_{1}(\theta)=1\right\}}\right\} \mathbf{1}_{\left\{\theta \notin\left(\beta^{0}, \beta^{1}\right)\right\}}\right] .
\end{aligned}
$$

Since

$$
\begin{aligned}
& P\left(\theta \in\left(\beta^{0}, \beta^{1}\right) \cap \hat{B}_{0} \mid \omega=1\right) \\
& =P\left(\theta \in\left(\beta^{0}, \beta^{1}\right) \cap B_{0} \mid \omega=1\right)+P\left(\theta \in D_{1} \mid \omega=1\right)-P\left(\theta \in D_{0} \mid \omega=1\right) \\
& <P\left(\theta \in\left(\beta^{0}, \beta^{1}\right) \cap B_{0} \mid \omega=1\right), \\
& P\left(\theta \in\left(\beta^{0}, \beta^{1}\right) \cap \hat{B}_{1} \mid \omega=1\right) \\
& =P\left(\theta \in\left(\beta^{0}, \beta^{1}\right) \cap B_{1} \mid \omega=1\right)+P\left(\theta \in D_{0} \mid \omega=1\right)-P\left(\theta \in D_{1} \mid \omega=1\right) \\
& >P\left(\theta \in\left(\beta^{0}, \beta^{1}\right) \cap B_{0} \mid \omega=1\right), \\
& P\left(\theta \in\left(\beta^{0}, \beta^{1}\right) \cap \hat{B}_{0} \mid \omega=0\right) \\
& =P\left(\theta \in\left(\beta^{0}, \beta^{1}\right) \cap B_{0} \mid \omega=0\right)+P\left(\theta \in D_{1} \mid \omega=0\right)-P\left(\theta \in D_{0} \mid \omega=0\right) \\
& =P\left(\theta \in\left(\beta^{0}, \beta^{1}\right) \cap B_{0} \mid \omega=0\right),
\end{aligned}
$$

the corresponding expression of $\pi(\hat{\sigma} \mid r, \hat{g})$ implies that we have $\pi_{1}(\sigma \mid r, g)<\pi_{1}(\hat{\sigma} \mid$ $r, \hat{g})$ when $0<u_{1}(1,0)-c_{1}<u_{1}(0,1)$.

We now show that (12) implies the existence of $\nu$ as claimed in the proposition. If $P\left(\theta \in\left(\beta^{0}, x\right) \cap B_{1}\right)>0$ for some $x>\beta^{0}$, then let $\nu=\inf \left\{x: x>\beta^{0}, P\left(\theta \in\left(\beta^{0}, x\right) \cap\right.\right.$ $\left.\left.B_{1}\right)>0\right\}$. By the definition of $\nu$, we have $P\left(\theta \in\left(\beta^{0}, \nu\right) \cap B_{1}\right)=\lim _{n \rightarrow \infty} P(\theta \in$ $\left.\left(\beta^{0}, \nu-\frac{1}{n}\right) \cap B_{1}\right)=0$. Furthermore, by (12), we have

$$
P\left(\theta \in\left(\nu+\frac{1}{n}, \beta^{1}\right) \cap B_{0}\right)=0 \text { for every } n=1,2, \ldots
$$

so that $P\left(\theta \in\left(\nu, \beta^{1}\right) \cap B_{0}\right)=\lim _{n \rightarrow \infty} P\left(\theta \in\left(\nu+\frac{1}{n}, \beta^{1}\right) \cap B_{0}\right)=0$. If $P\left(\left(\beta^{0}, x\right) \cap\right.$ $\left.B_{1}\right)=0$ for every $x \in\left(\beta^{0}, \beta^{1}\right)$, then let $\nu=\beta^{0}$, and if $P\left(\left(\beta^{0}, x\right) \cap B_{1}\right)>0$ for every $x \in\left(\beta^{0}, \beta^{1}\right)$, then let $\nu=\beta^{1}$. In each case, hence, we can conclude that if $g$ is optimal, then there exists $\nu \in\left[\beta^{0}, \beta^{1}\right]$ such that $g(\theta)=1$ for almost every $\theta \in\left(\nu, \beta^{1}\right)$ and $g(\theta)=0$ for almost every $\theta \in\left(\beta^{0}, \nu\right)$. 
Proof of Theorem 5 For $\sigma_{1}$ and $g$ specified in the theorem, we first verify that $\left(\sigma_{1}, \sigma_{2}^{*}\right)$ is an equilibrium under $(r, g)$ for any $r>0$ : $\sigma_{1}$ is a best response to $\sigma_{2}^{*}$ if

$$
E_{\omega}\left[u_{1}(1, g(\theta)) \mathbf{1}_{\{\omega=1\}} \mid \theta\right]-c_{1} \leq E_{\omega}\left[u_{1}(0, g(\theta)) \mathbf{1}_{\{\omega=1\}} \mid \theta\right] \text { for } \theta<\beta,
$$

and

$$
E_{\omega}\left[u_{1}(1, g(\theta)) \mathbf{1}_{\{\omega=1\}} \mid \theta\right]-c_{1} \geq E_{\omega}\left[u_{1}(0, g(\theta)) \mathbf{1}_{\{\omega=1\}} \mid \theta\right] \text { for } \theta \geq \beta,
$$

while $\sigma_{2}^{*}$ is a best response to $\sigma_{1}$ if

$$
\begin{aligned}
& E_{\omega, \theta}\left[u_{2}(0,1) \mathbf{1}_{\{\theta \in[\alpha, \beta), \omega=1\}} \mid g(\theta)=1\right]-c_{2} \\
& \geq E_{\omega, \theta}\left[u_{2}(0,0) \mathbf{1}_{\{\theta \in[\alpha, \beta), \omega=1\}} \mid g(\theta)=1\right],
\end{aligned}
$$

and

$$
\begin{aligned}
& E_{\omega, \theta}\left[u_{2}(1,0) \mathbf{1}_{\{\theta \in[\beta, \infty), \omega=1\}}+u_{2}(0,0) \mathbf{1}_{\{\theta \in(-\infty, \alpha), \omega=1\}} \mid g(\theta)=0\right] \\
& \geq E_{\omega, \theta}\left[u_{2}(1,1) \mathbf{1}_{\{\theta \in[\beta, \infty), \omega=1\}}+u_{2}(0,1) \mathbf{1}_{\{\theta \in(-\infty, \alpha), \omega=1\}} \mid g(\theta)=0\right]-c_{2} .
\end{aligned}
$$

It is clear from (7) that (15) and (16) hold for $\beta=\beta^{1}$. Moreover, (17) and (18) hold since $g(\theta)=1$ if and only if $\theta \in[\alpha, \beta)$, and by the definitions of $\alpha$ and $\beta$, we have

$$
E_{\omega}\left[u_{2}(0,1) \mathbf{1}_{\omega=1\}} \mid \theta\right]-c_{2} \geq E_{\omega}\left[u_{2}(0,0) \mathbf{1}_{\{\omega=1\}} \mid \theta\right]
$$

for every $\theta \in[\alpha, \beta)$,

$$
E_{\omega}\left[u_{2}(0,1) \mathbf{1}_{\omega=1\}} \mid \theta\right]-c_{2} \leq E_{\omega}\left[u_{2}(0,0) \mathbf{1}_{\{\omega=1\}} \mid \theta\right]
$$

for every $\theta \in(-\infty, \alpha)$, and

$$
E_{\omega}\left[u_{2}(1,1) \mathbf{1}_{\omega=1\}} \mid \theta\right]-c_{2} \leq E_{\omega}\left[u_{2}(1,0) \mathbf{1}_{\{\omega=1\}} \mid \theta\right]
$$

for every $\theta \in[\beta, \infty)$.

We now choose $r$ and evaluate the principal's equilibrium payoff under $(r, g)$. Let $\log$ denote the natural logarithm and $e=2.713 \ldots$ denote its base. Define

$$
\lambda=\frac{1}{2} \log \frac{\delta_{2}^{0}}{\delta_{1}^{1}}>0,
$$

and take $k>1$ such that

$$
\left(\frac{\delta_{2}^{0}}{\delta_{1}^{1}}\right)^{k}>e, \quad e^{\lambda\left(1-\frac{1}{k} e^{-2 \lambda-k}\right)}>\frac{\mu_{1}^{0}}{\mu_{1}^{0}-\delta_{1}^{0}-\delta_{1}^{1}}
$$


where the second inequality is possible by (9). Define $r>0$ by

$$
r=\left(\frac{p \delta_{1}^{1}}{1-p}\right)^{k}
$$

and $\alpha$ and $\beta$ as in the Theorem. By definition, we have

$$
\frac{f_{0}(\beta)}{f_{1}(\beta)}=\frac{p \delta_{1}^{1}}{1-p}=r^{\frac{1}{k}}
$$

and

$$
r \leq\left(\frac{\delta_{1}^{1}}{\delta_{2}^{0}}\right)^{k} \quad \Leftrightarrow \quad p \leq \frac{c_{2}}{d_{2}^{0}} .
$$

Under the assumption that $\epsilon$ has the standard normal distribution, it can also be verified that

$$
\beta=\frac{1}{2}-r^{2} \log \frac{p \delta_{1}^{1}}{1-p}=\frac{1}{2}-\frac{r^{2}}{k} \log r, \quad \text { and } \quad \alpha=\frac{1}{2}-r^{2} \log \frac{p \delta_{2}^{0}}{1-p}=\beta-2 \lambda r^{2} .
$$

The principal's ex ante expected payoff from $\sigma=\left(\sigma_{1}, \sigma_{2}^{*}\right)$ under $(r, g)$ equals

$$
\begin{aligned}
\pi_{1}(\sigma \mid r, g)=E_{\omega, \theta} & {\left[u_{1}(1,0) \mathbf{1}_{\{\omega=1, \theta \geq \beta\}}-c_{1} \mathbf{1}_{\{\theta \geq \beta\}}\right.} \\
& \left.+u_{1}(0,1) \mathbf{1}_{\{\omega=1, \alpha \leq \theta \in[\alpha, \beta)\}}+u_{1}(0,0) \mathbf{1}_{\{\omega=1, \theta<\alpha\}}\right] .
\end{aligned}
$$

Now define

$$
\begin{aligned}
\xi(r) & =\frac{\delta_{1}^{1}}{c_{1}(1-p)}\left\{\pi_{1}(\sigma \mid r, g)-\pi_{1}^{0}\right\} \\
& =r^{\frac{1}{k}}\left\{\left(\mu_{1}^{0}-\delta_{1}^{0}\right) F_{1}(\beta)-\mu_{1}^{0} F_{1}(\alpha)\right\}-\delta_{1}^{1}\left\{1-F_{0}(\beta)\right\} .
\end{aligned}
$$

Hence, $\xi(r)>0$ if and only if the principal's equilibrium payoff $\pi_{1}(\sigma \mid r, g)$ under $(r, g)$ strictly exceeds the level $\pi_{1}^{0}=p\left\{u(1,0)-c_{1}\right\}$ under perfect information. In what follows, we show that

$$
\xi^{\prime}(r) \geq 0 \text { for } r \leq\left(\frac{\delta_{1}^{1}}{\delta_{2}^{0}}\right)^{k},
$$

and that

$$
h \equiv \lim _{r \rightarrow 0} \frac{\xi(r)}{r^{\frac{1}{k}} F_{1}(\beta)}>0
$$

which would together imply that $\xi(r)>0$ for $0<r \leq\left(\frac{\delta_{1}^{1}}{\delta_{2}^{0}}\right)^{k}$. In view of $(22)$, this would prove the theorem. 
Note first that the following inequalities hold under (19):

$$
\begin{aligned}
& \frac{f_{1}(\beta)}{f_{1}(\alpha)}=e^{\lambda\left\{1+2 r^{2}\left(\lambda+\frac{1}{k} \log r\right)\right\}} \geq e^{\lambda\left(1-\frac{1}{k} e^{-2 \lambda-k}\right)}>\frac{\mu_{1}^{0}}{\mu_{1}^{0}-\delta_{1}^{0}-\delta_{1}^{1}}, \\
& \frac{F_{1}(\alpha)}{F_{1}(\beta)}=\frac{\int_{-\infty}^{\alpha} f_{1}(\theta) d \theta}{\int_{-\infty}^{\beta} f_{1}(\theta) d \theta}<\frac{f_{1}(\alpha)}{f_{1}(\beta)} .
\end{aligned}
$$

Differentiating $\xi$, we obtain

$$
\begin{aligned}
\xi^{\prime}(r) & =\left(\mu_{1}^{0}-\delta_{1}^{0}\right) F_{1}(\beta)-\mu_{1}^{0} F_{1}(\alpha) \\
& +r^{\frac{1}{k}+1}\left[\left(\mu_{1}^{0}-\delta_{1}^{0}\right) f_{1}(\beta)\left(\frac{\beta-1}{r}\right)^{\prime}-\mu_{1}^{0} f_{1}(\alpha)\left(\frac{\alpha-1}{r}\right)^{\prime}\right] \\
& +\delta_{1}^{1} r f_{0}(\beta)\left(\frac{\beta}{r}\right)^{\prime} .
\end{aligned}
$$

Using (27), we observe that

$$
\begin{aligned}
\left(\mu_{1}^{0}-\delta_{1}^{0}\right) F_{1}(\beta)-\mu_{1}^{0} F_{1}(\alpha) & =F_{1}(\beta)\left\{\mu_{1}^{0}-\delta_{1}^{0}-\mu_{1}^{0} \frac{F_{1}(\alpha)}{F_{1}(\beta)}\right\} \\
& >F_{1}(\beta)\left\{\mu_{1}^{0}-\delta_{1}^{0}-\mu_{1}^{0} \frac{f_{1}(\alpha)}{f_{1}(\beta)}\right\} \\
& >F_{1}(\beta)\left\{\mu_{1}^{0}-\delta_{1}^{0}-\mu_{1}^{0} \frac{\mu_{1}^{0}-\delta_{1}^{0}-\delta_{1}^{1}}{\mu_{1}^{0}}\right\} \\
& >0 .
\end{aligned}
$$

On the other hand, since $r<e^{-1}$ for $p \leq \frac{c_{2}}{d_{2}^{0}}$ by (20) and (22), we have

$$
\begin{aligned}
& \left(\frac{\beta-1}{r}\right)^{\prime}=\frac{1}{2 r^{2}}-\log r-1 \geq \frac{1}{2 r^{2}}, \\
& \left(\frac{\alpha-1}{r}\right)^{\prime}=\left(\frac{\beta-1}{r}\right)^{\prime}-2 \lambda, \\
& \left(\frac{\beta}{r}\right)^{\prime}=\left(\frac{\beta-1}{r}\right)^{\prime}-\frac{1}{r^{2}} .
\end{aligned}
$$

Using these as well as (21) and (27), we can evaluate $\xi^{\prime}$ as

$$
\begin{aligned}
\xi^{\prime}(p) & \geq r f_{0}(\beta) \\
& \times\left[\frac{1}{2 r^{2}}\left\{\mu_{1}^{0}-\delta_{1}^{0}+\delta_{1}^{1}-\frac{f_{1}(\alpha)}{f_{1}(\beta)} \mu_{1}^{0}\right\}+2 \lambda-\delta_{1}^{1} \frac{1}{r^{2}}\right] \\
& >r^{1+\frac{1}{k}} f_{1}(\beta) \frac{1}{2 r^{2}}\left\{\mu_{1}^{1}-\delta_{1}^{0}-\delta_{1}^{1}-\frac{f_{1}(\alpha)}{f_{1}(\beta)} \mu_{1}^{0}\right\} \\
& >0 .
\end{aligned}
$$


This shows (25). To prove (26), we rewrite

$$
\frac{\xi(r)}{r^{\frac{1}{k}} F_{1}(\beta)}=\frac{1}{\delta_{1}^{1}}\left\{\mu_{1}^{0}-\delta_{1}^{0}-\mu_{1}^{0} \frac{F_{1}(\alpha)}{F_{1}(\beta)}\right\}-\frac{1-F_{0}(\beta)}{r^{\frac{1}{k}} F_{1}(\beta)} .
$$

Lemma 7 , the definition of $\lambda$, and (9) together imply that

$$
\begin{aligned}
\lim _{r \rightarrow 0} \frac{\xi(r)}{r^{\frac{1}{k}} F_{1}(\beta)} & =\frac{1}{\delta_{1}^{1}}\left\{\mu_{1}^{0}-\delta_{1}^{0}-\mu_{1}^{0} e^{-\lambda}\right\}-1 \\
& >\frac{1}{\delta_{1}^{1}}\left\{\mu_{1}^{0}-\delta_{1}^{0}-\mu_{1}^{0} \frac{\mu_{1}^{0}-\delta_{1}^{0}-\delta_{1}^{1}}{\mu_{1}^{0}}\right\}-1=0 .
\end{aligned}
$$

This completes the proof.

\section{Lemma 7}

$$
\lim _{r \rightarrow 0} \frac{F_{1, r}(\alpha)}{F_{1, r}(\beta)}=e^{-\lambda}, \quad \lim _{r \rightarrow 0} \frac{1-F_{0, r}(\beta)}{r^{\frac{1}{k}} F_{1, r}(\beta)}=1,
$$

Proof. Note first that

$$
\begin{aligned}
& \lim _{r \rightarrow 0} r^{2}\left(\frac{\beta}{r}\right)^{\prime}=\lim _{r \rightarrow 0} r^{2}\left(\frac{\alpha}{r}\right)^{\prime}=-\frac{1}{2}, \\
& \lim _{r \rightarrow 0} r^{2}\left(\frac{\beta-1}{r}\right)^{\prime}=\lim _{r \rightarrow 0} r^{2}\left(\frac{\alpha-1}{r}\right)^{\prime}=\frac{1}{2} .
\end{aligned}
$$

We also have

$$
\lim _{r \rightarrow 0} \frac{f_{0}(\alpha)}{f_{0}(\beta)}=\lim _{r \rightarrow 0} e^{\frac{\beta^{2}-\alpha^{2}}{2 r^{2}}}=\lim _{r \rightarrow 0} e^{\lambda\left(2 \beta-2 \lambda r^{2}\right)}=e^{\lambda},
$$

and

$$
\lim _{r \rightarrow 0} \frac{F_{1}(\beta)}{f_{1}(\beta)}=\lim _{r \rightarrow 0} \frac{e^{-\frac{(\beta-1)^{2}}{2 r^{2}}}\left(\frac{\beta-1}{r}\right)^{\prime}}{e^{-\frac{(\beta-1)^{2}}{2 r^{2}}}(-1)\left(\frac{\beta-1}{r}\right)\left(\frac{\beta-1}{r}\right)^{\prime}}=\lim _{r \rightarrow 0} \frac{r}{1-\beta}=0 .
$$

For the first equation, we have

$$
\begin{aligned}
\lim _{r \rightarrow 0} \frac{F_{1, r}(\alpha)}{F_{1, r}(\beta)} & =\lim _{r \rightarrow 0} \frac{F\left(\frac{\alpha-1}{r}\right)}{F\left(\frac{\beta-1}{r}\right)}=\lim _{r \rightarrow 0} \frac{f\left(\frac{\alpha-1}{r}\right)\left(\frac{\alpha-1}{r}\right)^{\prime}}{f\left(\frac{\beta-1}{r}\right)\left(\frac{\beta-1}{r}\right)^{\prime}} \\
& =\lim _{r \rightarrow 0} e^{\frac{(\beta-1)^{2}-(\alpha-1)^{2}}{2 r^{2}}} \frac{r^{2}\left(\frac{\alpha-1}{r}\right)^{\prime}}{r^{2}\left(\frac{\beta-1}{r}\right)^{\prime}} \\
& =\lim _{r \rightarrow 0} e^{\lambda\left(2 \beta-2-2 \lambda r^{2}\right)}=e^{-\lambda},
\end{aligned}
$$


where the second equality follows from L'Hospital's rule, and the next to the last equality uses (28). For the second equation, we have by L'Hospital's rule,

$$
\begin{aligned}
\lim _{r \rightarrow 0} \frac{1-F_{0, r}(\beta)}{r^{\frac{1}{k}} F_{1, r}(\beta)} & =\lim _{r \rightarrow 0} \frac{1-F\left(\frac{\beta}{r}\right)}{r^{\frac{1}{k}} F\left(\frac{\beta-1}{r}\right)} \\
& =\lim _{r \rightarrow 0} \frac{-r f_{0}(\beta)\left(\frac{\beta}{r}\right)^{\prime}}{\frac{1}{k} r^{\frac{1}{k}-1} F_{1}(\beta)+r^{\frac{1}{k}+1} f_{1}(\beta)\left(\frac{\beta-1}{r}\right)^{\prime}} \\
& =\lim _{r \rightarrow 0} \frac{-r \frac{f_{0}(\beta)}{f_{1}(\beta)}\left(\frac{\beta}{r}\right)^{\prime}}{\frac{1}{k} r^{\frac{1}{k}-1} \frac{F_{1}(\beta)}{f_{1}(\beta)}+r^{\frac{1}{k}+1}\left(\frac{\beta-1}{r}\right)^{\prime}} \\
& =\lim _{r \rightarrow 0} \frac{-r^{2}\left(\frac{\beta}{r}\right)^{\prime}}{\frac{1}{k} \frac{F_{1}(\beta)}{f_{1}(\beta)}+r^{2}\left(\frac{\beta-1}{r}\right)^{\prime}} \\
& =\frac{1 / 2}{1 / 2}=1,
\end{aligned}
$$

where the fourth equality follows from (21) and the fifth from (28), (29) and (30).

Proof of Proposition 6 For $\alpha \in \boldsymbol{R}$, let $(r, g)$ and $\sigma=\left(\sigma_{1}, \sigma_{2}^{*}\right)$ be such that $r_{p}=0$ and $r_{q}>0$, and

$$
\left(\sigma_{1}(\theta), g(\theta)\right)= \begin{cases}(1,1) & \text { if } \theta_{p}=1 \text { and } \theta_{q} \geq \alpha, \\ (1,0) & \text { if } \theta_{p}=1 \text { and } \theta_{q}<\alpha, \\ (0,0) & \text { otherwise. }\end{cases}
$$

Then the principal's ex ante expected payoff under $\sigma$ and $(r, g)$ is given by

$$
\begin{aligned}
& \pi_{1}(\sigma \mid r, g) \\
& =p\left[u_{1}(1,1)\left\{q P\left(\theta_{q} \geq \alpha \mid \omega_{q}=1\right)+(1-q) P\left(\theta_{q} \geq \alpha \mid \omega_{q}=0\right)\right\}\right. \\
& \left.\quad+u_{1}(1,0)\left\{q P\left(\theta_{q}<\alpha \mid \omega_{q}=1\right)+(1-q) P\left(\theta_{q}<\alpha \mid \omega_{q}=0\right)\right\}-c_{1}\right] .
\end{aligned}
$$

Hence

$$
\pi_{1}(\sigma \mid r, g)-\pi_{1}^{0}=\operatorname{pm}_{1}^{1}\left\{-q P\left(\theta_{q}<\alpha \mid \omega_{q}=1\right)+(1-q) P\left(\theta_{q} \geq \alpha \mid \omega_{q}=0\right)\right\},
$$

where $m_{1}^{1}=u_{1}(1,1)-u_{1}(1,0)$. Therefore, $(r, g)$ dominates perfect information if

$$
\frac{P\left(\theta_{q}<\alpha \mid \omega_{q}=1\right)}{P\left(\theta_{q} \geq \alpha \mid \omega_{q}=0\right)}<\frac{1-q}{q} .
$$


On the other hand, the agent's incentive conditions are written as

$$
P(\omega=(1,1) \mid g(\theta)=1) \delta_{2}^{1}(1)+P(\omega=(1,0) \mid g(\theta)=1) \delta_{2}^{1}(0) \geq c_{2},
$$

when he receives the advice $g(\theta)=1$, and

$$
P(\omega=(1,1) \mid g(\theta)=0) \delta_{2}^{1}(1)+P(\omega=(1,0) \mid g(\theta)=0) \delta_{2}^{1}(0) \leq c_{2},
$$

when he receives the advice $g(\theta)=0$. Since $q \delta_{2}^{1}(1)+(1-q) \delta_{2}^{1}(0)<c_{2}$ by our assumption on $q$, if (32) holds, then (33) must hold as well. Using

$$
P\left(\omega_{q}=1 \mid \theta_{q} \geq \alpha\right)=\frac{q P\left(\theta_{q} \geq \alpha \mid \omega_{q}=1\right)}{q P\left(\theta_{q} \geq \alpha \mid \omega_{q}=1\right)+(1-q) P\left(\theta_{q} \geq \alpha \mid \omega_{q}=0\right)},
$$

we can rewrite (32) as

$$
\frac{P\left(\theta_{q} \geq \alpha \mid \omega_{q}=1\right)}{P\left(\theta_{q} \geq \alpha \mid \omega_{q}=0\right)} \geq \frac{1-q}{q} \frac{c_{2}-d_{2}^{1}(0)}{d_{2}^{1}(1)-c_{2}}
$$

Now note that

$$
\frac{P\left(\theta_{q}<\alpha \mid \omega_{q}=1\right)}{P\left(\theta_{q} \geq \alpha \mid \omega_{q}=0\right)} \text { is increasing in } r, \text { and } \rightarrow 0 \text { as } r \rightarrow 0 \text { when } \alpha<\frac{1}{2},
$$

and that

$$
\frac{P\left(\theta_{q} \geq \alpha \mid \omega_{q}=1\right)}{P\left(\theta_{q} \geq \alpha \mid \omega_{q}=0\right)} \text { is decreasing in } r, \text { and } \rightarrow \infty \text { as } r \rightarrow 0
$$

Hence, if we let $\alpha<1 / 2$ and take $r>0$ sufficiently small, then they satisfy both (31) and (34).

\section{References}

[1] Helmut Bester and Roland Strausz (2001), "Contracting with imperfect commitment and the revelation principle: The single agent case," Econometrica, 69, 1077-98.

[2] David S. Brookshire, Mark A. Thayer, John Tschirhart, and William D. Schulze (1985), "A Test of the Expected Utility Model: Evidence from Earthquake Risks," Journal of Political Economy, 93(2), 369-389.

[3] Juan D. Carrillo and Thomas Mariotti (2000), "Strategic Ignorance as a SelfDisciplining Device," Review of Economic Studies, 67(3), 529-44. 
[4] Jacques Cremer (1995), "Arm's Length Relationship," Quarterly Journal of Economics, 110(2), 275-295.

[5] Mattias Dewatripont and Eric Maskin (1994), "Contractual Contingencies and Renegotiation," Rand Journal of Economics, 26(4), 704-19.

[6] Sara L. Eggers, and Baruch Fischhoff (2004), "Setting Policies for Consumer Communications: A Behavioral Decision Theory Approach," Journal of Public Policy and Marketing, 23(1), 14-27.

[7] Baruch Fischhoff (1994), "What Forecasts (Seem to) Mean," International Journal of Forecasting, 10, 387-403.

[8] Baruch Fischhoff (2011), "Communicating about the Risk of Terrorism (or Anything Else)," American Psychologist, 66(6), 520-31.

[9] Jack Hirschleifer (1971), "The Private and Social Value of Information and the Reward from Incentive Activity," American Economic Review, 61, 561-74.

[10] Charles W. Howe and Harold C. Cochrane (1976), "A Decision Model for Adjusting to Natural Hazard Events with Application to Urban Snow Storms," Review of Economics and Statistics, 58, 50-58.

[11] Anke S. Kessler (1998), "The Value of Ignorance," Rand Journal of Economics, $29(2), 339-54$.

[12] Stephen Morris and Hyun-Song Shin (2002), "Social Value of Public Information," American Economic Review, 92, 1521-34.

[13] Richard R. Nelson and Sidney G. Winter (1964), "A Case Study in Economics of Information and Coordination: The Weather Forecasting System," Quarterly Journal of Economics, 78, 420-441.

[14] Makoto Saito (2008), "Social Science of Earthquake Forecasting," Shosai-noMado 580, 12-24 (in Japanese).

[15] Emmanuel Skoufias (2003), "Economic Crises and Natural Disasters: Coping Strategies and Policy Implications," World Development, 31(3), 1087-1101.

[16] Vasiliki Skreta (2006), "Sequentially Optimal Mechanisms," Review of Economic Studies, 73, 1085-1111. 
[17] Lars, E. O. Svensson (2006), "Social Value of Public Information: Morris and Shin (2002) is Actually Pro Transparency, Not Con," American Economic Review, 96, 448-51.

[18] Carl E. Walsh (2007), "Announcements and the Role of Policy Guidance," working paper, University of California, Santa Cruz. 